International Mathematical Forum, 2, 2007, no. 33, 1647 - 1656

\title{
Weighted Composition Followed by Differentiation between Bergman Spaces
}

\author{
Ajay K. Sharma ${ }^{1}$ \\ School of Applied Physics and Mathematics \\ Shri Mata Vaishno Devi University \\ P/O Kakryal, Udhampur-182121, India \\ aksju_76@yahoo.com \\ Som Datt Sharma and Sanjay Kumar \\ Department of Mathematics \\ University of Jammu-180006, India \\ somdatt_jammu@yahoo.co.in
}

\begin{abstract}
In this paper we consider linear operators $M_{\psi} C_{\varphi} D$ and $M_{\psi} D C_{\varphi}$ acting between weighted Bergman spaces, where $M_{\psi}, C_{\varphi}$ and $D$ are multiplication, composition and differentation operators respectively. Our goal is to characterize those holomorphic self maps $\varphi$ of $\mathbb{D}$ for which $M_{\psi} D C_{\varphi}$ and $M_{\psi} C_{\varphi} D$ acts boundedly and compactly between weighted Bergman spaces.
\end{abstract}

Mathematics Subject Classification: Primary 47B33, 46E10; Secondary 30D 55

Keywords: composition operator, multiplication operator, weighted composition operator, differentation, generalized Nevanlinna counting function, vanishing Carleson measure, weighted Bergman spaces

\section{Introduction}

Thoughout this paper we denote by $H(\mathbb{D})$, the space of holomorphic functions on $\mathbb{D}$, where $\mathbb{D}$ is the open unit disk in the complex plane $\mathbb{C}$. Let $\psi$ and $\varphi$ be

\footnotetext{
${ }^{1}$ Supported by CSIR-grant ( F.No. 9/100(100)2002 EMR-1).
} 
holomorphic maps on $\mathbb{D}$ such that $\varphi(\mathbb{D}) \subset \mathbb{D}$, we can define a linear operator $\psi C_{\varphi}$ on $H(\mathbb{D})$, called weighted composition by

$$
\psi C_{\varphi} f=\psi(f \circ \varphi)
$$

The operator $\psi C_{\varphi}$ can be regarded as a generalization of a multiplication operator and a composition operator, In case $\psi \equiv 1$ or $\varphi(z)=z, \psi C_{\varphi}$ reduces to the composition operator $C_{\varphi}$ or the multiplication operator $M_{\psi}$, respectively. For general back ground on composition operators, we refer [CoM 95] and [Sh 93] and references therein

Weighted composition operators appear naturally in different contexts. For example, Singh and Sharma [SiS 79] related the boundedness of composition operators on Hardy space of the upper half-plane with the boundedness of weighted composition operators on the Hardy space of the open unit disk $\mathbb{D}$. Isometries in many Banach spaces of analytic functions are just weighted composition operators, for example see [Fo 64].

Recently, several authors have studied weighted composition operators on different spaces of analytic functions. For example, one can refer to $[\mathbf{C o H} 04]$ for study of these operators on Hardy spaces, [Ka 79] and [OhT 01] for disk algebra, [OhZ 01] and [OSZ 03] for Bloch-type spaces and [MiS 97] for study of these operators on Bergman spaces.

In this paper we consider linear operators $M_{\psi} C_{\varphi} D$ and $M_{\psi} D C_{\varphi}$ acting between weighted Bergman spaces, where $M_{\psi}, C_{\varphi}$ and $D$ are multiplication, composition and differentation operators respectively. Our goal is to characterize those holomorphic self maps $\varphi$ of $\mathbb{D}$ for which $M_{\psi} D C_{\varphi}$ and $M_{\psi} C_{\varphi} D$ acts boundedly and compactly between weighted Bergman spaces.

\section{Preliminaries}

For $\alpha>-1$, the weighted Bergman space $A_{\alpha}^{p}$, is the set of analytic functions on the disk with

$$
\|f\|_{A_{\alpha}^{p}}^{p}=\int_{\mathbb{D}}|f(z)|^{p} d \lambda_{\alpha}(z)<\infty
$$

where $d \lambda_{\alpha}(z)=(\alpha+1)\left(1-|z|^{2}\right)^{\alpha} d A(z)$ and $d A(z)=d x d y / \pi=r d r d \theta / \pi, z=$ $x+i y \in \mathbb{D}$. The following sharp estimate tells us how fast an arbitrary function from $A_{\alpha}^{p}$ grow near the boundary. Let $f \in A_{\alpha}^{p}$. Then for every $z$ in $\mathbb{D}$, we have

$$
|f(z)| \leqslant \frac{\|f\|_{A_{\alpha}^{p}}}{\left(1-|z|^{2}\right)^{(2+\alpha) / p}}
$$

with equality if and only if $f$ is a constant multiple of the function

$$
k_{a}(z)=\left(\frac{1-|z|^{2}}{(1-\bar{a} z)^{2}}\right)^{2+\alpha / p}
$$


It can be easily shown that $\left\|k_{a}\right\|_{A_{\alpha}^{p}} \approx 1$ with constant depending only on $\alpha$ and $p$ [Sm 96, page 400]. For general background of weighted Bergman spaces $A_{\alpha}^{p}$ and weighted Bloch spaces, one may consult $[\mathbf{Z h ~ 9 0 ]}$ and $[\mathbf{H K Z ~ 0 0 ] ~ a n d ~}$ the references therein.

In what follows we make extensive use of Carleson measure techniques, so we give a short introduction to Carleson sets and Carleson measures. For a point $\zeta$ on the boundary of $\mathbb{D}$ we define the Carleson set

$$
S(\zeta, \delta)=\{z \in \mathbb{D}:|\zeta-z|<\delta\}
$$

We use Carleson sets along with a more convinient choice of pseudohyperbolic disks. For $0<r<1$ and $a \in \mathbb{D}$, denote by $D(a, r)$, the disk whose pseudohyperbolic center is $a$ and whose pseudohyperbolic radius is $r$ :

$$
D(a, r)=\left\{z \in \mathbb{D}:\left|\frac{a-z}{1-\bar{a} z}\right|<r\right\}
$$

The notation $|D(a, r)|_{A}$ will denote the area of $D(a, r)$. For fixed $0<r<1$ the area of $D(a, r)$ has the estimation:

$$
|D(a, r)|_{A} \approx\left(1-|a|^{2}\right)^{2} \approx\left(1-|z|^{2}\right)^{2} \approx|D(z, r)|_{A}
$$

for $z \in D(a, r)$, where $\approx$ means that the two quantities are bounded above and below by the constants independent of $a$. For fixed $0<r<1$, it is also known that for $z \in D(a, r)$,

$$
|1-\bar{a} z| \approx\left(1-|a|^{2}\right)
$$

Also for each $D(a, r)$, there is a $\zeta \in \partial \mathbb{D}$ so that $D(a, r) \subset S(\delta, \zeta)$ for $\delta \approx 1-|a|$. A positive Borel measure $\mu$ on $\mathbb{D}$ is called $\alpha$-Carleson measure if

$$
\sup _{\delta>0} \sup _{\zeta \in \partial \mathbb{D}} \frac{\mu(S(\delta, \zeta))}{\delta^{\alpha+2}}<\infty
$$

and it will be called a vanishing Carleson measure if in addition

$$
\lim _{\delta \longrightarrow 0} \sup _{\zeta, \in \delta D} \frac{\mu(S(\zeta, \delta))}{\delta^{\alpha+2}}=0 .
$$

\section{Boundedness and Compactness of $M_{\psi} D C_{\varphi}$}

In this section, we characterize those holomorphic self-maps of $\mathbb{D}$ for which $M_{\psi} D C_{\varphi}$ maps $A_{\alpha}^{p}$ boundedly and compactly into $A_{\beta}^{q}$.

To do so we need a generalized Nevanlinna counting function, which will be required for the change of variable. 
Definition 3.1. Let $\varphi$ and $\psi$ be holomorphic self-maps of $\mathbb{D}$ such that $\varphi(\mathbb{D}) \subset \mathbb{D}$. Let $q \geq 1$ and $\beta>-1$. For $w \in \mathbb{D}, w \neq 0$, we define

$$
N_{\psi, \varphi}^{q, \beta}(w)=\sum|\psi(z)|^{q}\left|\varphi^{\prime}(z)\right|^{q-2}\left(\log \frac{1}{|z|}\right)^{\beta}
$$

where sum extends over all solutions of $\varphi(z)=w$ and we name it generalized Nevanlinna counting function.

We need a generalized change of variable formula. In the following formula, $\left\{z_{j}(w)\right\}$ denote the sequence of zeros of $\varphi(z)-w$ repeated according to multiplicity.

Theorem 3.2. [CoM 95] If $g$ and $W$ are non-negative measurable functions on $\mathbb{D}$, then

$$
\int_{\mathbb{D}} g(\varphi(z))\left|\varphi^{\prime}(z)\right|^{2} W(z) d A(z)=\int_{\varphi(\mathbb{D})} g(\varphi(z))\left(\sum_{j \geq 1} W\left(z_{j}(w)\right)\right) d A(w) .
$$

We assume from now on that $r \in(0,1)$ is fixed.

Theorem 3.3. Let $1 \leqslant p \leqslant q$, and $\alpha, \beta>-1$. Let $\varphi$ and $\psi$ be a holomorphic maps and $\mathbb{D}$ such that $\varphi(\mathbb{D}) \subset \mathbb{D}$ and $\psi \varphi^{\prime} \in A_{\beta}^{q}$. Let $d \mu(w)=$ $N_{\psi, \varphi}^{q, \beta}(w) d A(w)$. Then the following are equivalent:

(1) $M_{\psi} D C_{\varphi}$ maps $A_{\alpha}^{p}$ boundedly into $A_{\beta}^{q}$.

(2) $\mu(D(a, r))=O\left(\left(1-|a|^{2}\right)^{q(\alpha+2+p) / p}\right)$ as $|a| \rightarrow 1$.

In order to prove the Theorem 3.3 we need following result of Luecking $[\mathbf{L u}$ 85 ] in which he characterized positive measures $\mu$ with the property:

$$
\left\|f^{(n)}\right\|_{L^{q}(\mu)} \leqslant C\|f\|_{A_{\alpha}^{p}}
$$

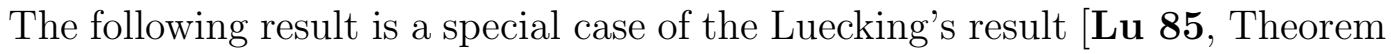
$2.2]$ for $n=1$ in case $1 \leqslant p \leqslant q$.

Theorem 3.4. Let $1 \leqslant p \leqslant q$, and $\alpha, \beta>-1$. Let $\mu$ be a finite positive measure on $\mathbb{D}$. Then the following are equivalent:

(2) $\quad \mu(D(a, r))=O\left(\left(1-|a|^{2}\right)^{q(\alpha+2+p) / p}\right)$ as $|a| \rightarrow 1$.

For the case $1 \leqslant q<p$, Luecking used Khinchine's inequality and other estimates to obtain a version of Theorem 3.4 for $f^{(n)}$, where $f \in A^{p}$.

We are interested in the case $\mathrm{n}=1$ and $f \in A_{\alpha}^{p}$.

The following result is a slight modification of Luecking's result (see $[\mathbf{H i P} \mathbf{0 5}$ ] also).

Theorem 3.5. Let $1 \leqslant q<p$, and $\alpha>-1$. Let $\mu$ be a finite positive measure on $\mathbb{D}$. Let $\Omega(z)=\left(1-|z|^{2}\right)^{-(\alpha+2+q)} \mu(D(z, r))$. Then the following are equivalent:

(1) $\quad\left\|f^{\prime}\right\|_{L^{q}(\mu)} \leqslant C\|f\|_{A_{\alpha}^{p}}$ for all $f \in A_{\alpha}^{p}$ 
(2) $\Omega \in L^{p / p-q}\left(\nu_{\alpha}\right)$.

We are now ready to prove Theorem 3.3.

Proof of Theorem 3.3. Since $\psi \varphi^{\prime} \in A_{\beta}^{q}$, change of variable formula 3.2 implies that $\mu$ is a finite measure. Thus Theorem 3.4 applies.

Note that

$$
\begin{aligned}
\left\|M_{\psi} D C_{\varphi} f\right\|_{A_{\beta}^{q}}^{q} & \approx \int_{\mathbb{D}}\left|f^{\prime}(\varphi(z))\right|^{q}|\psi(z)|^{q}\left|\varphi^{\prime}(z)\right|^{q}\left(\log \frac{1}{|z|}\right)^{\beta} d A(z) \\
& =\int_{\mathbb{D}}\left|f^{\prime}(w)\right|^{q} N_{\psi, \varphi}^{q, \beta}(w) d A(w) \\
& =\left\|f^{\prime}\right\|_{L^{q}(\mu)}^{q} .
\end{aligned}
$$

Since $M_{\psi} D C_{\varphi}$ maps $A_{\alpha}^{p}$ boundedly into $A_{\beta}^{q}$, so we have

$$
\left\|f^{\prime}\right\|_{L^{q}(\mu)}^{q}=\left\|M_{\psi} D C_{\varphi} f\right\|_{A_{\beta}^{q}}^{q} \leqslant C\|f\|_{A_{\alpha}^{p}}
$$

for all $f \in A_{\alpha}^{p}$. Hence Theorem 3.4 implies that

$$
\mu(D(a, r))=O\left(\left(1-|a|^{2}\right)^{q(\alpha+2+p) \mid p} \text { as }|a| \rightarrow 1 .\right.
$$

Conversely, suppose that (ii) holds. Then by Theorem 3.4, we have

$$
\left\|M_{\psi} D C_{\varphi} f\right\|_{A_{\beta}^{q}}=\left\|f^{\prime}\right\|_{L^{q}(\mu)} \leqslant C\|f\|_{A_{\alpha}^{p}}
$$

and hence $M_{\psi} C_{\varphi} D$ maps $A_{\alpha}^{p}$ boundedly into $A_{\beta}^{q}$.

Theorem 3.6: Let $1 \leqslant p \leqslant q$, and $\alpha, \beta>-1$. Let $\varphi$ and $\psi$ be holomorphic maps on $\mathbb{D}$ such that $\varphi(\mathbb{D}) \subset \mathbb{D}$ and $\psi \varphi^{\prime} \in A_{\beta}^{q}$. Let $d \mu(w)=N_{\psi, \varphi}^{q, \beta}(w) d A(w)$. Then the following are equivalent:

(1) $\quad M_{\psi} D C_{\varphi}$ maps $A_{\alpha}^{p}$ compactly into $A_{\beta}^{q}$.

(2) $\mu(D(a, r))=o\left(\left(1-|a|^{2}\right)^{q(\alpha+2+p) / p}\right)$ as $|a| \rightarrow 1$.

Proof: First suppose that $M_{\psi} D C_{\varphi}$ maps $A_{\alpha}^{p}$ compactly into $A_{\beta}^{q}$. Let $a \in \mathbb{D}$ and consider the function

$$
f_{a}(z)=\frac{\left(1-|a|^{2}\right)^{(\alpha+2) / p}}{(1-\bar{a} z)^{2(\alpha+2) / p}} .
$$

Clearly $\left\|f_{a}\right\|_{A_{\alpha}^{p}} \approx 1$ and $f_{a}$ converges to zero uniformly on compact subsets of $\mathbb{D}$ as $|a| \rightarrow 1$. Since $M_{\psi} D C_{\varphi}$ maps $A_{\alpha}^{p}$ compactly into $A_{\beta}^{q}$, so for gives $\epsilon>0$, we can find $r_{0}, 0<r_{0}<1$ such that $\left\|M_{\psi} D C_{\varphi} f_{a}\right\|_{A_{\alpha}^{p}}<\epsilon$ for $|a|>r_{0}$.

Thus

$$
\epsilon>\int_{\mathbb{D}}\left|f_{a}^{\prime}(z)\right|^{q} d \mu(z) \geq \int_{D(a, r)}\left|f_{a}^{\prime}(z)\right|^{q} d \mu(z)
$$

for $|a|>r_{0}$. Since for $z \in D(a, r)$,

$$
\left|f_{a}^{\prime}(z)\right| \approx \frac{1}{\left(1-|a|^{2}\right)^{(\alpha+2+p) / p}}
$$


and so above estimate yields

$$
\mu(D(a, r))<\epsilon\left(\left(1-|a|^{2}\right)^{q(\alpha+2+p) / p}\right.
$$

for all $a$ with $|a|>r_{0}$. Hence

$$
\mu(D(a, r))=o\left(\left(1-|a|^{2}\right)^{q(\alpha+2+p) / p)} \text { as }|a| \rightarrow 1 .\right.
$$

Conversely, assume that (ii) holds and let $\left\{f_{n}\right\}$ be a sequence in $A_{\alpha}^{p}$ such that $\left\|f_{n}\right\|_{A_{\alpha}^{q}} \leqslant M$ and $f_{n} \rightarrow 0$ uniformly on compact subsets of $\mathbb{D}$. To show that $M_{\psi} D C_{\varphi}$ maps $A_{\alpha}^{p}$ compactly into $A_{\beta}^{q}$, it is sufficient to show that

$$
\left\|M_{\psi} D C_{\varphi} f_{n}\right\|_{A_{\beta}^{q}}^{q}=\left\|f_{n}^{\prime}\right\|_{L^{q}(\mu)}^{q} \rightarrow 0 \text { as } n \rightarrow \infty
$$

By a standard estimate of Luecking [Lu 93], page 338, we have

$$
\left\|M_{\psi} D C_{\varphi} f_{n}\right\|_{A_{\beta}^{q}}^{q} \leqslant C \int_{\mathbb{D}} \frac{1}{\left(1-|a|^{2}\right)^{2+q}} \int_{D(a, r)}\left|f_{n}(z)\right|^{q} d A(z) d \mu(a) .
$$

Note that

$$
\chi_{D(a, r)}(z)=\chi_{D(z, r)}(a) \text { and } 1-|a|^{2} \approx 1-|z|^{2}
$$

for $a \in D(z, r)$. Also by 2.1, we have

$$
\left|f_{n}(z)\right| \leqslant C \frac{\left\|f_{n}\right\|_{A_{\alpha}^{p}}}{\left(1-|z|^{2}\right)^{(2+\alpha) / p}} .
$$

By an application of Fubini's theorem, we get

$$
\begin{aligned}
\left\|M_{\psi} D C_{\varphi} f_{n}\right\|_{A_{\beta}^{q}}^{q} & \leqslant C^{\prime} \int_{\mathbb{D}}\left|f_{n}(z)\right|^{q} \frac{\mu(D(z, r))}{\left(1-|z|^{2}\right)^{2+q}} d A(z) \\
& \leqslant C^{\prime}|| f_{n} \|_{A_{\alpha}^{q}}^{q-p} \int_{\mathbb{D}}\left|f_{n}(z)\right|^{p} \frac{\mu(D(z, r))}{\left(1-|z|^{2}\right)^{(\alpha q+2 q-p q-\alpha p) / p}} d A(z) \\
& \leqslant C^{\prime} M^{q-p}\left(\int_{|z| \leqslant r_{0}}\left|f_{n}(z)\right|^{p} \frac{\mu(D(z, r))}{\left(1-|z|^{2}\right)^{(\alpha q+2 q+p q-\alpha p) / p}} d A(z)\right. \\
& \left.+\int_{|z|>r_{0}}\left|f_{n}(z)\right|^{p} \frac{\mu(D(z, r))}{\left(1-|z|^{2}\right)^{(\alpha q+2 q+p q-\alpha p) / p}} d A(z)\right) \\
& =I+I I .
\end{aligned}
$$

Now (ii) implies that for a given $\epsilon>0$, we can find $r_{0}, 0<r_{0}<1$ such that

$$
\begin{aligned}
I I & =C^{\prime} M^{q-p} \int_{|z|>r_{0}}\left|f_{n}(z)\right|^{p} \frac{\mu(D(z, r))}{\left(1-|z|^{2}\right)^{(\alpha q+2 q+p q-\alpha p) / p}} d A(z) \\
& \leqslant \epsilon C^{\prime} M^{q-p} \int_{|z|>r_{0}}\left|f_{n}(z)\right|^{p}\left(1-|z|^{2}\right)^{\alpha} d A(z) \\
& \leqslant \epsilon C^{\prime} M^{q-p}\left\|f_{n}\right\|_{A_{\alpha}^{p}}^{p} \\
& \leqslant \epsilon C^{\prime} M^{q} .
\end{aligned}
$$


Since $f_{n} \rightarrow 0$ uniformly on compact subsets of $\mathbb{D}$

$$
\begin{aligned}
I & =C^{\prime} M^{q-p} \int_{|z| \leqslant r_{0}}\left|f_{n}(z)\right|^{p} \frac{\mu(D(z, r))}{\left(1-|z|^{2}\right)^{(\alpha q+2 q+p q-\alpha p) / p}} d A(z) \\
& \leqslant C_{1} C^{\prime} M^{q-p} \epsilon \int_{\mathbb{D}} \mu(D(z, r)) d A(z) \\
& \leqslant C_{1} C_{2} C^{\prime} M^{q-p} \epsilon \int_{\mathbb{D}} \mu(\mathbb{D}) d A(z) \\
& =C_{1} C_{2} C_{3} C^{\prime} M^{q-p} \epsilon
\end{aligned}
$$

for $n$ large enough. Thus

$$
\lim _{n \rightarrow \infty}\left\|M_{\psi} D C_{\varphi} f_{n}\right\|_{A_{\beta}^{q}}^{q}=0,
$$

and hence $M_{\psi} D C_{\varphi}$ maps $A_{\alpha}^{p}$ compactly into $A_{\beta}^{q}$.

Theorem 3.7 Let $1 \leqslant p<q$, and $\alpha, \beta>-1$. Let $d \mu(z)=N_{\psi, \varphi}^{q, \beta} d A(z)$ and let $\psi \varphi^{\prime} \in A_{\beta}^{q}$. Let $G(z)=\left(1-|z|^{2}\right)^{-(\alpha+q+2)} \mu(D(a, r))$. Then the following are equivalent:

(1) $M_{\psi} D C_{\varphi}$ maps $A_{\alpha}^{p}$ boundedly into $A_{\beta}^{q}$.

(2) $M_{\psi} D C_{\varphi}$ maps $A_{\alpha}^{p}$ compactly into $A_{\beta}^{q}$.

(3) $G \in L^{p /(p-q)}\left(\nu_{\alpha}\right)$.

Proof. (1) $\Leftrightarrow$ (3) Suppose (1) holds. By change of variable formula as in Theorem 3.2, we have

$$
\left\|M_{\psi} D C_{\varphi} f\right\|_{A_{\beta}^{q}}^{q}=\left\|f^{\prime}\right\|_{L^{q}(\mu)}^{q}
$$

Since $M_{\psi} D C_{\varphi}$ maps $A_{\alpha}^{p}$ boundedly into $A_{\beta}^{q}$, we can find a positive constant $\mathrm{C}$ such that

$$
\left\|f^{\prime}\right\|_{L^{q}(\mu)}=\left\|M_{\psi} D C_{\varphi} f\right\|_{A_{\beta}^{q}} \leqslant C\|f\|_{A_{\alpha}^{p}}
$$

and so by Theorem $3.4, M_{\psi} D C_{\varphi}$ maps $A_{\alpha}^{p}$ boundedly into $A_{\beta}^{q}$ if and only if $G \in L^{p /(p-q)}\left(\nu_{\alpha)}\right.$.

It is clear that (2) implies (1).

It remains to verify that (3) implies (2). Assume that

$$
\left\|f_{n}\right\|_{A_{\alpha}^{p}} \leqslant C
$$

and $f_{n} \rightarrow 0$ uniformly on compact subsets of $\mathbb{D}$. It is sufficient to show that

$$
\lim _{n \rightarrow \infty}\left\|M_{\psi} D C_{\varphi} f_{n}\right\|_{A_{\beta}^{q}}=0 .
$$

As in the proof of the Theorem 3.5, we have

$$
\left\|M_{\psi} D C_{\varphi} f_{n}\right\|_{A_{\beta}^{q}} \leqslant C \int_{\mathbb{D}}\left|f_{n}(z)\right|^{q} G(z) d \nu_{\alpha}(z) .
$$


Let $\epsilon>0$. Then the hypothesis on $G$ implies that there exists $r_{0}, 0<r_{0}<1$, with the property

$$
\int_{|z|>r_{0}} G^{p / p-q}(z) d \nu_{\alpha}(z)<\epsilon^{p / p-q} .
$$

It follows by Holder's inequality that

$$
\begin{aligned}
\int_{|z|>r_{0}}\left|f_{n}(z)\right|^{q} G(z) d \nu_{\alpha}(z) & \\
& \leqslant\left(\int_{\mathbb{D}}\left|f_{n}(z)\right|^{p} d \nu_{\alpha}\right)^{q / p}\left(\int_{|z|>r_{0}} G^{p / p-q} d \nu_{\alpha}(z)\right)^{(p-q) / p} \\
& \leqslant \epsilon\left\|f_{n}\right\|^{q} A_{\alpha}^{p} \\
& \leqslant C \epsilon .
\end{aligned}
$$

Thus we have

$$
\int_{|z|>r_{0}}\left|f_{n}(z)\right|^{q} G(z) d \nu_{\alpha}(z) \leqslant C \epsilon .
$$

Since $f_{n} \rightarrow 0$ uniformly on compact subsets of $\mathbb{D}$, so $\left|f_{n}(z)\right|<\epsilon$ for all $z$ such that $|z|<r_{0}$ and for all $n \geq n_{0}$. Thus

$$
\int_{|z| \leqslant r_{0}}\left|f_{n}(z)\right|^{q} G(z) d \nu_{\alpha}(z) \leqslant \epsilon^{q} \int_{|z| \leqslant r_{0}} G(z) d \nu_{\alpha}(z)
$$

for $n \geq n_{0}$. Since $\psi \varphi^{\prime} \in A_{\beta}^{q}$,

$$
G(z) \leqslant C \mu(D(z, r)) \leqslant C \mu(\mathbb{D})<\infty
$$

and thus

$$
\int_{|z| \leqslant r_{0}} G(z) d \nu_{\alpha}(z) \leqslant C \int_{\mathbb{D}} \mu(D(z, r)) d \nu_{\alpha}(z) \leqslant C .
$$

Thus

$$
\int_{|z| \leqslant r}\left|f_{n}(z)\right|^{q} G(z) d \nu_{\alpha}(z) \leqslant C \epsilon
$$

for $n \geq n_{0}$. Hence $M_{\psi} D C_{\varphi}$ maps $A_{\alpha}^{p}$ compactly into $A_{\beta}^{q}$.

\section{Boundedness and Compactness of $M_{\psi} C_{\varphi} D$}

Now we discuss boundedness and compactness of the operator $M_{\psi} C_{\varphi} D$ acting between weighted Bergman spaces. Proofs follow exactly on same lines, so we omit the details.

Theorem 4.1. Let $1 \leqslant p \leqslant q<\infty$, and $\alpha, \beta>-1$. Let $\varphi$ and $\psi$ be holomorphic maps on $\mathbb{D}$ such that $\varphi(\mathbb{D}) \subset \mathbb{D}$ and $\psi \varphi \in A_{\beta}^{p}$. Then the following are equivalent:

(1) $\quad M_{\psi} C_{\varphi} D$ maps $A_{\alpha}^{p}$ boundedly into $A_{\beta}^{q}$ 
(2) $\left(\mu_{\beta} \circ \varphi^{-1}\right)(D(a, r))=O\left(\left(1-|a|^{2}\right)^{q(a+2+p) / p}\right)$ as $|a| \rightarrow 1$.

where $\mu_{\beta} \circ \varphi^{-1}$ is the pull-back measure induced by $\nu_{\beta}$. Here $d \mu_{\beta}(z)=|\psi(z)|^{q} d \nu_{\beta}(z)$.

Proof: First suppose that $M_{\psi} C_{\varphi} D$ maps $A_{\alpha}^{p}$ boundedly into $A_{\beta}^{q}$. Since $\psi \varphi \in A_{\beta}^{q}$, change of variable formula of measure theory ( see [Ha 74], Theorem $\mathrm{C}$, page 173) implies that $\mu_{\beta} \circ \varphi^{-1}$ is a finite measure

$$
\begin{aligned}
\left\|M_{\psi} C_{\varphi} D f\right\|_{A_{\beta}^{q}}^{q} & =\int_{\mathbb{D}}\left|f^{\prime}(\varphi(z))\right|^{q}|\psi(z)|^{q} d \nu_{\beta}(z) \\
& =\int_{\mathbb{D}}\left|f^{\prime}(z)\right|^{q} d\left(\mu_{\beta} \circ \varphi^{-1}\right)(z) \\
& =\left\|f^{\prime}\right\|_{L^{q}\left(\mu_{\beta} \circ \varphi^{-1}\right)}^{q},
\end{aligned}
$$

Since $M_{\psi} C_{\varphi} D$ maps $A_{\alpha}^{p}$ boundedly into $A_{\beta}^{q}$, so we have

$$
\begin{aligned}
\left\|f^{\prime}\right\|_{L^{q}(\mu)}^{q} & =\left\|M_{\psi} C_{\varphi} D f\right\|_{A_{\beta}^{q}}^{q} \\
& \leqslant C\|f\|_{A_{\beta}^{q}}^{q}
\end{aligned}
$$

for all $f \in A_{\beta}^{q}$. Hence Theorem 4.2.4 implies that

$$
\left(\mu_{\beta} \circ \varphi^{-1}\right)(D(a, r))=O\left(\left(1-|a|^{2}\right)^{q(\alpha+2+p) \mid p} \text { as }|a| \rightarrow 1 .\right.
$$

Conversely suppose that (ii) holds. Then by Theorem 3.4, we have

$$
\left\|M_{\psi} C_{\varphi} D f\right\|_{A_{\beta}^{q}}=\left\|f^{\prime}\right\|_{L^{q}\left(\mu_{\beta} \circ \varphi^{-1}\right)} \leqslant C\|f\|_{A_{\alpha}^{p}}
$$

and hence $M_{\psi} C_{\varphi} D$ maps $A_{\alpha}^{p}$ boundedly into $A_{\beta}^{q}$.

Theorem 4.2. Let $1 \leqslant p \leqslant q$, and $\alpha, \beta>-1$. Let $\varphi$ and $\psi$ be holomorphic maps on $\mathbb{D}$ such that $\varphi(\mathbb{D}) \subset \mathbb{D}$ and $\psi \varphi \in A_{\beta}^{q}$. Then the following are equivalent.

(1) $M_{\psi} C_{\varphi} D$ maps $A_{\alpha}^{p}$ compactly into $A_{\beta}^{q}$

(2) $\left(\mu_{\beta} \circ \varphi^{-1}\right)(D(a, r))=O\left(\left(1-|a|^{2}\right)^{q(a+2+p) / p}\right)$ as $|a| \rightarrow 1$.

Theorem 4.3. Let $1 \leqslant p<p$, and $\alpha, \beta>-1$. Let $\varphi$ and $\psi$ be holomorphic maps on $\mathbb{D}$ such that $\varphi(\mathbb{D}) \subset \mathbb{D}$ and $\psi \varphi \in A_{\beta}^{q}$ and let $\Phi(z)=$ $\left(1-|z|^{2}\right)^{-(\alpha+2+q)}\left(\mu_{\beta} \circ \varphi^{-1}\right)(D(z, r))$. Then the following are equivalent.

(1) $M_{\psi} C_{\varphi} D$ maps $A_{\alpha}^{p}$ boundedly into $A_{\beta}^{q}$

(2) $M_{\psi} C_{\varphi} D$ maps $A_{\alpha}^{p}$ compactly into $A_{\beta}^{q}$

(3) $\Phi \in L^{p / p-q}\left(\nu_{\alpha}\right)$

\section{References}

[CoH 04] M. D. Contreras and A. G. Hernandez-Diaz, 'Weighted composition operators on spaces of functions with derivative in a Hardy space', J. Operator Theory, 52 (2004), 173-184. 
[CoM 95] C. C. Cowen and B. D. MacCluer, 'Composition operators on spaces of analytic functions', CRC Press Boca Raton, New York, 1995.

[CuZ 04] Z. Cuckovic and R. Zhao, 'Weighted composition operators on the Bergman space', J. London Math. Soc., 70 (2004), 499-511.

[Fo 64] F. Forelli, 'The isometries of $H^{p}$ spaces', Canad. J Math., 16 (1964) 721-728.

[Ha 74] P. R. Halmos, Measure theory, Springer-Verlag, New York, 1974.

[HiP 05] R.A. Hibschweiler and N. Portnoy, 'Composition followed by differentiation between Bergman and Hardy spaces' Rocky Mountain Journal of Mathematics 35 2005, 843-855.

[HKZ 00] H. Hedenmalm, B. Korenblum and K. Zhu, 'Theory of Bergman spaces', Springer, New York, Berlin, etc. 2000.

[Ka 79] H. Kamowitz, 'Compact operators of the form $u C_{\varphi}$,' Pacific J. Math. , 80 (1979), 205-211.

[Lu 85] D. H. Luecking, 'Forward and reverse Carleson inequalities for functions in Bergman spaces and their derivatives,' Amer.. Math. J. 40 1985, 85-111.

[Lu 85] D. H. Luecking, 'Embedding theorems for spaces of analytic functions via Khinchine's inequality,' Mich. Math. J. 40 1993, 333-358.

[MiS 97] G. Mirzakarimi and K. Seddighi, 'Weighted composition operators on Bergman and Dirichlet spaces', Georgian. Math. J., 4 (1997), 373-383.

[OhT 01] S. Ohno and H. Takagi, 'Some properties of weighted composition operators on algebras of analytic functions', J. Nonlinear Convex Anal. , 2 (2001), 369-380.

[OhZ 01] S. Ohno and R. Zhao, 'Weighted composition operators on the Bloch space,' Bull. Austral. Math. Soc., 63 (2001), 177-185.

[OSZ 03] S. Ohno, K. Stroethoff and R. Zhao, 'Weighted composition operators between Bloch-type spaces', Rocky Mountain J. Math., 33 (2003), 191-215.

[Sh 93] J. H. Shapiro, 'Composition operators and classical function theory', SpringerVerlag, New York. 1993.

[SiS 79] R. K. Singh and S. D. Sharma, 'Composition operators on a functional Hilbert space',Bull. Austral. Math. Soc. 20(1979), 377-384.

[Sm 96] W. Smith, 'Composition operators between Bergman and Hardy spaces', Trans. Amer. Math. Soc, 348 (1996), 2331-2348.

[Zh 90] K. Zhu, 'Operator theory in function spaces', Marcel Dekker, New York, 1990.

Received: October 11, 2006 